\title{
Pressure-Induced Molecular Absorption in Stellar Atmospheres
}

\author{
Aleksandra Borysow
}

Physics Department, Michigan Technological University

Houghton, MI 49931, USA

\section{The Nature of Collision-induced Absorption}

Pressure-induced absorption arises in complexes of two or more inert atoms or molecules, due to dipole moments induced during the collisional interaction. The term "pressure-induced" still prevails in the astrophysical literature, yet "collision-induced" absorption (CIA), or "interaction-induced" absorption seems more appropriate and is commonly used elsewhere. Ordinary absorption processes in the infrared arise from individual, polar molecules interacting with electromagnetic radiation. As a consequence, the intensity of the allowed lines increases linearly with density. CIA, on the other hand, is most striking in gases composed of nonpolar, infrared-inactive molecules. Induced spectral lines are observed at rovibrational frequencies which are dipole-forbidden in single (i.e. non-interacting) molecules. Dipole transitions may, however, be induced in the interacting pair. The new symmetry of the electronic cloud of a collisional complex may be very different from those of the isolated molecules and thus commonly allows for a transient dipole, which then interacts with radiation. Collision-induced absorption increases quadratically in the low density limit, thus reflecting the two-body origin of the basic absorption process. At higher gas densities, ternary interactions become significant and cubic and higher-order contributions to the observable absorption are then commonly seen.

A few characteristic features of CIA will be discussed next. Interactioninduced lines are generally very broad, an immediate consequence of the fact that induced dipoles last only for a short time, roughly the mean time of a collision, $t_{c}$, which depends on temperature and reduced mass of the interacting pair. For hydrogen at room temperature, the approximate (effective) time of the duration of a collision inducing a dipole amounts to roughly $5 \cdot 10^{-14} \mathrm{~s}$, which leads to a spectral width, $\Delta \nu \approx 1 / t_{c}$, of approximately $600 \mathrm{~cm}^{-1}$. At higher temperatures, naturally, the widths will be even larger (at $3000 \mathrm{~K}, t_{c} \approx 10^{-14} \mathrm{~s}$, and $\Delta \nu \approx 3000 \mathrm{~cm}^{-1}$ ). In general, CIA spectral widths follow approximately a $\Delta \nu \propto T^{1 / 2}$ dependence ( $T$ being the temperature).

Two mechanisms are mainly responsible for the induction of a dipole in molecular encounters. Electronic overlap induction exists in atomic and molec- 
ular pairs at short distances. It is a purely quantum effect caused by the Pauli principle: due to the overlap of the electronic clouds, some states become inaccessible to electrons. As a result, the redistribution of the electronic charge occurs and a transient dipole is being induced in a pair at close distances (roughly smaller than $3 \AA$ ). The other mechanism is a multipolar induction which, to a large extent, is a classical process characteristic of the molecular gases. The electric fields surrounding any molecule may be represented by a superposition of multipolar fields. For example, the lowest, non-vanishing multipole moment of $\mathrm{H}_{2}$ is the quadrupole, or that of $\mathrm{CH}_{4}$ is the octopole. A collisional partner in the electric multipolar field of one molecule is polarized and thus interacts with radiation. Multipolar induction is the dominant induction mechanism for most common molecules, except the ones having small polarizabilities. Classical multipole induction provides a rather accurate approximation of the actual induced dipole function at large intermolecular distances (those larger than $3 \AA$ or so).

Based on these simple facts, one is able to explain the structure of the CIA spectra of molecular pairs. Figure 1 shows a typical spectrum of $\mathrm{H}_{2}$ pairs at room temperature. The spectral intensities marked on the $y$-axis are those corresponding to one amagat ${ }^{1}$ of $\mathrm{H}_{2}$. For a given number density of a gas, one needs to multiply the plotted absorption spectrum by the number of amagats squared. The frequencies are given in $\mathrm{cm}^{-1}$ units. Broad induced absorption

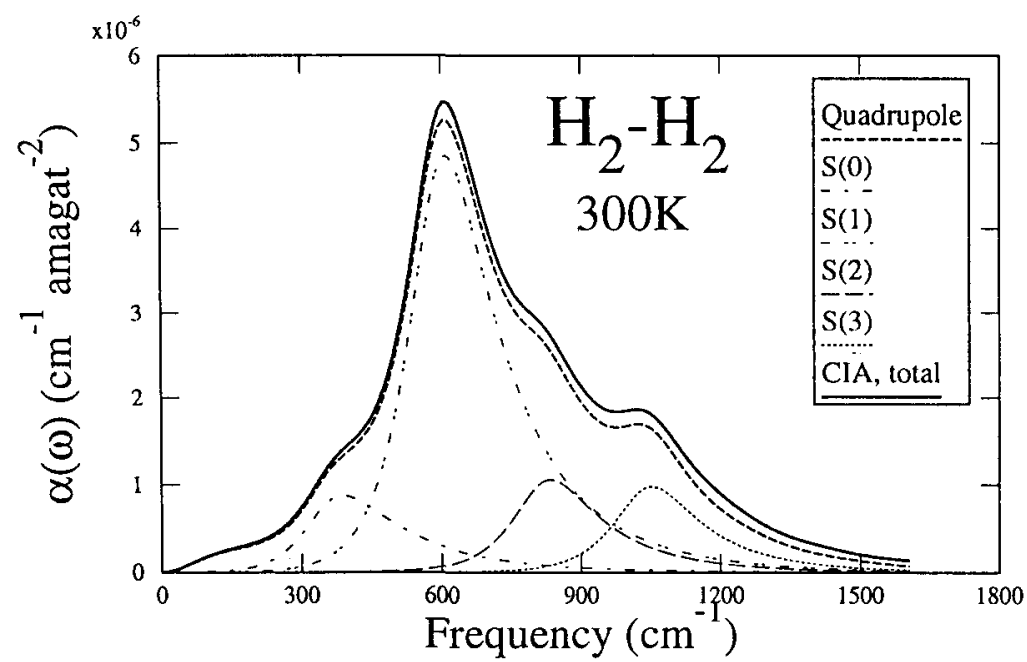

Fig. 1. Rotranslational collision-induced absorption spectrum of $\mathrm{H}_{2}-\mathrm{H}_{2}$ at $300 \mathrm{~K}$ (solid line). Dashed line denotes the spectral contribution due to the quadrupolar induction. $S(J)$ denote lines due to separate rotational transitions, $J \rightarrow J+2$ for populated rotational states $J=0,1,2,3$.

11 amagat corresponds very nearly to the number density of gas under normal conditions, and is equal to $2.687 \cdot 10^{19} \mathrm{~cm}^{-3}$. 
lines appear at the rotational transition frequencies of the hydrogen molecule, mainly with $J \rightarrow J+2$ transitions ( $S$ lines).

To illustrate this, let us first imagine that a dipole is induced during a collision between two (different) atoms. At large separations, the dipole will be initially zero. With decreasing interatomic distance, the dipole will increase as the electronic clouds begin to overlap. The dipole strength will reach a maximum near the point of the closest approach. After that, it will fall off to zero again as the separation increases. On the whole, the strength of the induced dipole will be significant only during the "collision". The Fourier transform of the autocorrelation function of this dipole function $\boldsymbol{\mu}(t)$ will give rise to a broad spectral density profile, which is centered at zero frequency, commonly called a purely "translational" spectrum.

Next, let us briefly consider the case of two interacting molecules. The dipole now largely arises from multipole induction. If for the moment we focus on $\mathrm{H}_{2}-\mathrm{X}$ collisions ( $\mathrm{X}$ may be a helium atom or another hydrogen molecule, or indeed any other nonpolar species), the quadrupolar field of a rotating hydrogen molecule will introduce an additional modulation of the time-dependent induced dipole function, $\boldsymbol{\mu}(t)$, which will result in the appearance of a similar, broad "translational" spectral profile, only shifted from zero frequency to the rotational transition frequencies of the $\mathrm{H}_{2}$ molecule $(S(J)$ lines, Fig. 1). This simplified model does, in fact, describe the rotranslational (RT) CIA spectra quite well. What must be understood, is that it is a hydrogen molecule which undergoes rotational transitions corresponding to the $S(J)$ lines. The presence of the collisional (interacting) partner is essential for the appearance of these rotational lines, which otherwise are dipole-forbidden in a single $\mathrm{H}_{2}$ molecule. The simultaneous change of the orbital and rotational angular momenta taking place during the collision, make the rotational transitions temporarily "allowed". Each rotational transition in hydrogen molecule, is accompanied by an absorption (or emission) between the translational states of a collisional pair. It results in the the "translational" broadening of the $S(J)$ profiles.

If we also consider the possibility of vibration of a hydrogen molecule, the vibrational transition frequency introduces additional oscillations of the induced dipole, causing a diffuse line to appear also at the rovibrational transition frequency of the hydrogen molecule (rovibrational CIA spectra). In this case, all three degrees of freedom participate in the absorption process, translation, rotation and vibration. After the collision, the hydrogen molecule is found in a higher rotation-vibration state. Summarizing, we will expect CIA spectra to cover the entire infrared region, from the "purely translational" (centered at zero frequency), to the rotranslational (Fig. 1) and the rovibrational and even the overtone bands. The latter ones will appear around $v \rightarrow v^{\prime}$ transitions, with $v, v^{\prime}=0,1,2,3 \ldots$ At low temperatures only the ground vibrational level is populated, so $v=0$, and the transitions to $v^{\prime}=1$ (the fundamental band) and $v^{\prime}=2,3, \ldots$ (the first and higher overtones) are observed. In principle, at elevated temperatures, a non-zero population of higher $v$ states will give rise to the absorption "hot bands" which, however, have not yet been observed in the 


\section{laboratory.}

We are concerned with hydrogen and helium, the major components of stellar atmospheres. To complete the picture, in Fig. 2 we show the RT spectrum of $\mathrm{H}_{2}-$ He pairs at the temperature of $300 \mathrm{~K}$. Even though quadrupolar induction clearly takes place (just as in pure $\mathrm{H}_{2}$ gas), the spectral profile looks quite different. The dashed line marks the quadrupolar term. However, another spectral component, absent in Fig. 1, is shown here (dotted line): the so-called "isotropic overlap". This term, which is independent of the molecular orientations, is zero for the $\mathrm{H}_{2}-\mathrm{H}_{2} \mathrm{RT}$ spectra, due to symmetries inconsistent with a dipole moment. This essential difference between the $\mathrm{H}_{2}-\mathrm{H}_{2}$ and $\mathrm{H}_{2}-\mathrm{He}$ spectra persists also more or less in the rovibrational spectra. We will refer to this difference when we consider the various CIA models proposed in the past.

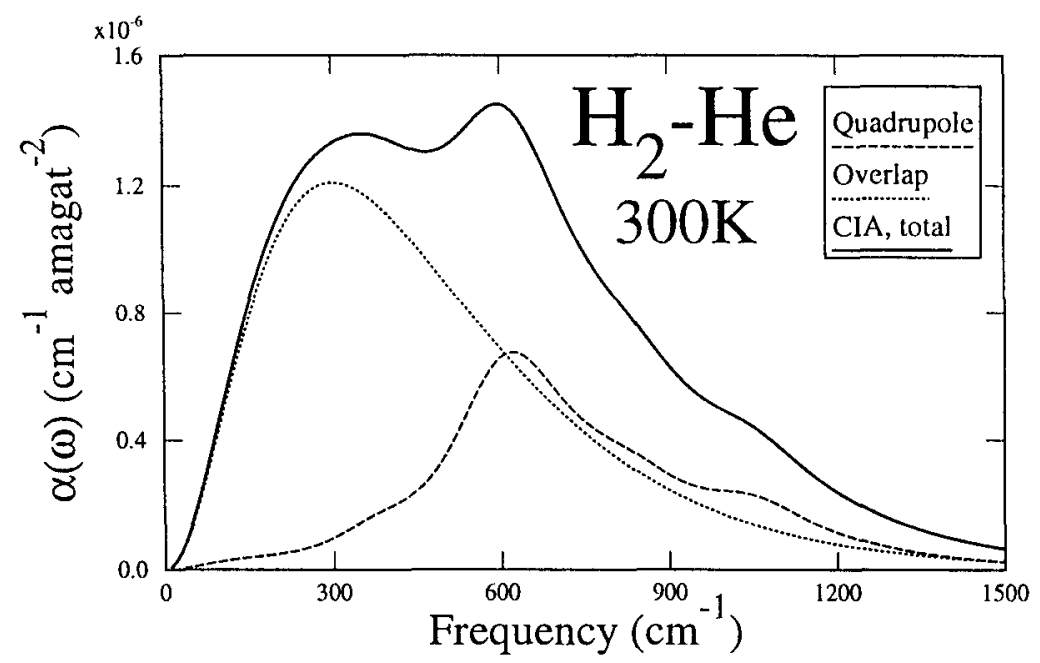

Fig. 2. Rotranslational collision-induced absorption spectrum of $\mathrm{H}_{2}-\mathrm{He}$ at $300 \mathrm{~K}$ (solid line). The dashed line marks the spectral contribution due to quadrupolar induction and the dotted line marks the contribution due to isotropic overlap induction.

Collision-induced spectra are an intrinsic property of dense matter. In fluids that are not too dense, the induced spectra may be represented in the form of a series in powers of the gas density, $\varrho^{n}$. For nonpolar gases, the binary spectra, i.e., the coefficient of the $\varrho^{2}$ term, will be the first non vanishing term. At low densities this absorption will hardly be measurable, yet with increasing density it may become quite striking and the three-, four-, etc., body contributions may also be discernible. For hydrogen and helium, predominantly binary spectra are observed at densities below 10 amagats, except at a few frequencies where the intercollisional process affects the profiles. The characteristic "intercollisional dip" has been observed in the rovibrational CIA spectra, and has been explained by van Kranendonk (1968) as a many-body process, which is due to the destructive 
interference of the radiation process occurring during collisions. This interference effect is due to the negative correlations existing between the dipole moments induced in consecutive collisions. The main "dip" appears at the $Q(J)$ branch $(\Delta(J)=0, \Delta(v) \neq 0)$, but weaker intercollisional dips have also been measured at the $S(J)(\Delta(J)=2)$ lines. The dip, unlike the CIA spectra, broadens linearly with the increasing density of the gas and its intensity does not follow the $\rho^{2}$ dependence. An excellent review paper, covering all the details of this phenomenon, which is not part of the binary CIA discussed here, has been written by Lewis (1985).

As mentioned above, the basic CIA induction process is "weak". Induced dipole strengths are roughly two orders of magnitude smaller than those of typical molecules like $\mathrm{H}_{2} \mathrm{O}$ or $\mathrm{HCl}$. Since the intensity is proportional to the dipole moment squared, CIA may typically be rather negligible in the presence of allowed dipole absorption unless the gas density is high and/or the metallicity (i.e. abundance of elements heavier than helium) is low. In cool, dense stellar atmospheres of low-metallicity stars, composed basically of hydrogen and helium, CIA may dominate the opacity at low (i.e. infrared) frequencies, owing to the absence of dipole moments which are not interaction-induced.

Finally, we mention another important property of CIA spectra. Unlike the well known pressure- broadened line profiles, where the spectral width increases with increasing density, CIA profiles are generally independent of density unless gas densities are very high. That means that a variation of density amounts to a simple rescaling of the profiles, by a factor proportional to density squared. The shape depends on the temperature of the gas and the reduced mass of the collisional partners, i.e. on factors which determine the time scale of molecular encounters. Of course, it also depends on the intermolecular potential and the induced dipole function.

For more details concerning collision-induced absorption, the reader is referred to the review papers by Borysow et al. (1987, 1991), Borysow \& Frommhold (1985), and various other contributions to the same conference proceedings (i.e., Birnbaum et al. 1984) or related proceeedings edited by Szudy (1989) and Frommhold \& Keto (1990). The first monograph on the subject (Frommhold $1993)$ is due to appear this year.

\section{Stellar Applications}

It is well-known that collision-induced absorption plays a significant role in the atmospheres of cool stars, such as white dwarfs, brown dwarfs, M dwarfs and the hypothetical Population III stars. In general, CIA will be important in all cool, zero-metallicity stellar environments. The term "cool" is meant to imply low densities of free electrons, i.e. largely neutral environments where molecules exist. Below, we will give a brief historical review of how CIA became recognized as a significant source of opacity in the various stellar atmospheres.

The importance of pressure-induced absorption for the opacity of the cool stars has been first studied by Linsky (1969), following Trafton's (1964) pioneer- 
ing study on planetary atmospheres. He pointed out that sources of continuous opacity may influence the radiation field more strongly than the molecular bands, because most radiative energy ("flux") escapes between the individual lines, i.e. where the absorption is weakest. Linsky's focus has been on late-type stars, with temperatures lower than $4000 \mathrm{~K}$ and rather high densities, between $10^{-3}$ and 10 amagats (pressures from 0.01 to 100 atmospheres). He demonstrated that at temperatures below $2500 \mathrm{~K}$ practically all hydrogen exists in the molecular form, $\mathrm{H}_{2}$. Therefore, molecular hydrogen plays an important role in the atmospheric (infrared) opacity. He provided the first models of CIA spectra of $\mathrm{H}_{2}-\mathrm{H}_{2}$ and $\mathrm{H}_{2}-\mathrm{He}$ at temperatures ranging from 600 to $4000 \mathrm{~K}$. At that time, no accurate computations of CIA existed; good experimental results at such temperatures are even now unavailable for $\mathrm{H}_{2}-\mathrm{H}_{2}$ and $\mathrm{H}_{2}-\mathrm{He}$ systems. The models have been based on Trafton's (1966) early quantum mechanical computations for $\mathrm{H}_{2}-\mathrm{H}_{2}$ pairs at temperatures below $600 \mathrm{~K}$. Linsky's model was based on semiempirical extrapolation procedures. The associated uncertainty of these results could only recently be determined in quantitative terms, when exact quantum calculations became available. Similar work for $\mathrm{H}_{2}-\mathrm{He}$ has been even more uncertain: the spectral profiles were assumed to be identical to those of pure $\mathrm{H}_{2}$ pairs, and the intensity was rescaled by some empirical factor. Figure 3 compares the quantum mechanical models of the RT CIA computed for the $\mathrm{H}_{2}-\mathrm{H}_{2}$ and $\mathrm{H}_{2}-\mathrm{He}$ systems at the temperature of $3000 \mathrm{~K}$. The figure demonstrates the significant difference between the shapes of the two spectra at $3000 \mathrm{~K}$, yet the difference is significant at all temperatures (compare also Figs. 1 and 2). Despite its shortcomings,

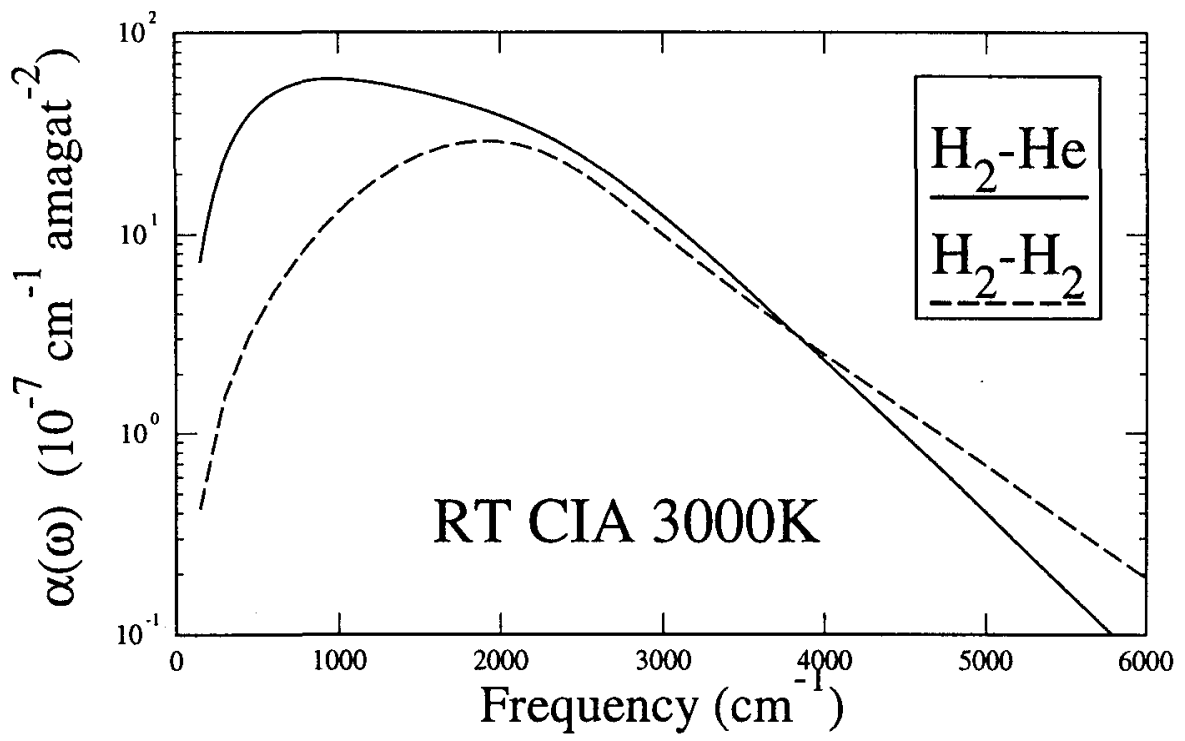

Fig. 3. Rotranslational collision-induced absorption spectra of $\mathrm{H}_{2}-\mathrm{H}_{2}$ and $\mathrm{H}_{2}-\mathrm{He}$ at 3000 K. Computations based on models by Borysow et al. (1988) and Borysow (1993). 
Linsky's work unmistakably pointed out the significance of collision-induced absorption for stellar atmospheres. Linsky concluded that at temperatures as low as $2500 \mathrm{~K}$, CIA is the primary source of the continuous opacity. Linsky's model profiles of high temperature CIA have been used for two decades by scientists modelling the cool, hydrogen-rich atmospheres. They enabled astronomers to get an independent confirmation of the importance of CIA in various stellar environments and presented a real motivation toward the development of more realistic models. Advanced, new computations, and their comparison with Linsky's data will be discussed in Sec. 4 .

At about the same time, Tsuji (1969) considered molecular opacities in cool stellar atmospheres, including various sources of continuous opacity. He estimated the rovibrational CIA spectrum of hydrogen pairs at $3000 \mathrm{~K}$ in the fundamental band of $\mathrm{H}_{2}$ using a simple, semiempirical procedure. Nonetheless, he has reached independently the same conclusion that CIA is an important source of opacity at high pressures, and that model atmospheres developed without accounting for it are likely to result in unreliable effective temperatures.

In the following decade collision-induced absorption in stellar atmospheres attracted more attention. Shipman (1977), working on the atmospheres of cool white dwarfs, has computed the first model atmosphere which, except for absorption sources accounted for before, also included Linsky's models. He considered pure hydrogen atmospheres at temperatures between 4000 and $8000 \mathrm{~K}$. His primary goal was to determine the role $\mathrm{H}_{2}$ molecules play in these atmospheres. $\mathrm{He}$ found that at a temperature of $4000 \mathrm{~K}$ CIA contributes essentially all the opacity at such wavelengths where the flux is emitted! At $5000 \mathrm{~K}$ CIA provided only $10 \%$ of the total opacity, on account of the larger abundance of electrons and ions, giving rise to the relatively much stronger opacities. These findings have significant implications: depending on whether CIA is included or not, different conclusions may be reached regarding the temperatures, radii and masses of cool stars. Mould \& Liebert (1978) in their paper on the atmospheric composition of cool white dwarfs have included CIA due to $\mathrm{H}_{2}-\mathrm{H}_{2}$ and $\mathrm{H}_{2}-\mathrm{He}$ pairs, using again Linsky's data. They computed new atmospheric models at temperatures between 4000 and $7000 \mathrm{~K}$. The overall importance of CIA is not explicitly stated, but the question regarding the reliability of Linsky's predictions was raised. The fact that no experimental data existed to test these estimates has been a serious drawback of any analysis making use of these results.

Population III stars are hypothetical first-generation, zero-metallicity stars, assumed to have formed in the early stages of the Galaxy formation. The stars are assumed to have condensed out of primordial matter and are therefore mainly composed of hydrogen and helium. At temperatures from 1000 to $7000 \mathrm{~K}$, CIA may constitute the major source of opacity in such stars. Palla (1985) pointed out, for the first time, that the more conventional opacity sources, e.g., Rayleigh scattering by $\mathrm{H}, \mathrm{He}$ and $\mathrm{H}_{2}, e^{-}$scattering, bound-free and free-free absorption by $\mathrm{H}, \mathrm{H}^{-}$, and $\mathrm{H}_{2}^{+}$, are insufficient. When CIA was finally included for the computations of the Rosseland mean opacities, the magnitude of the error of omitting CIA of $\mathrm{H}_{2}-\mathrm{H}_{2}, \mathrm{H}_{2}-\mathrm{He}$ was striking (Palla 1985). Whereas at high temperatures, 
typically above $3500 \mathrm{~K}$, CIA plays a minor role and other opacity sources dominate, CIA accounts for all the opacity at the lower temperatures. To be more specific, at the lowest temperature considered $(1500 \mathrm{~K})$ the difference between the Rosseland mean, computed with and without accounting for CIA, amounted to more than one order of magnitude at a density of $10^{-6}$ amagats, more than three orders of magnitude at $\rho=10^{-3}$ amagats, and up to five orders of magnitude at the highest densities considered ( $\rho=0.1$ amagats). In Palla's work, atmospheres composed of hydrogen $(71 \%)$ and helium $(28 \%)$ were considered and Linsky's (1969) data were again input. One year later, Stahler et al. (1986) confirmed Palla's findings. The conclusion was now made even stronger: at all densities between $10^{-8}$ and 0.1 amagats, and temperatures below $2000 \mathrm{~K}$, CIA has been found to be the dominant process of all continuous opacity sources accounted for, such as electron scattering, Rayleigh molecular scattering, $\mathrm{H}^{-}$ absorption and CIA. At higher temperatures, up to $3000 \mathrm{~K}$, CIA remains the dominant process at the higher densities. In other words, the source of opacity due to collision induced absorption cannot be neglected. Surprisingly enough, it has been demonstrated that, in the absence of other sources of opacity, CIA may still dominate the atmospheric absorption at densities as low as $10^{-8}$ amagats (it needs to be recalled that $\alpha(\omega) \propto \rho^{2}$ !).

A few years later, Lenzuni et al. (1991) presented a detailed analysis of zerometallicity stars. They considered the temperatures between 1000 and $7000 \mathrm{~K}$ and densities from $10^{-8}$ to $10^{3}$ amagats. The Rosseland mean, and the monochromatic opacities have been computed, which included collision-induced absorption of $\mathrm{H}_{2}-\mathrm{H}_{2}$ and $\mathrm{H}_{2}-\mathrm{He}$ pairs. For the first time models based on quantum mechanical computations were used in the analysis (for $\mathrm{H}_{2}-\mathrm{H}_{2}$ in the fundamental band: Borysow \& Frommhold 1990, and for all rovibrational bands of $\mathrm{H}_{2}-\mathrm{He}$ : Borysow et al. 1989, Borysow \& Frommhold 1989). The remainder of the hydrogen bands has been taken either from Linsky, or modeled independently by the authors. We mention that in the case of the RT hydrogen band an incorrect extrapolation of a low temperature model has been used for $\mathrm{H}_{2}-\mathrm{H}_{2}$ spectra. Despite these shortcomings, the authors demonstrated in an impressive way how CIA depends upon both temperature and density. For a fixed temperature at high enough density, CIA may become the major opacity source. At fixed density and low enough temperatures, more or less the same effect is achieved. CIA manifests itself mainly at frequencies below $15,000 \mathrm{~cm}^{-1}$. Additionally, it has been pointed out that accurate data concerning the first and possibly the second overtone may be of the utmost importance in the Rosseland mean opacity because, when CIA is dominant, the monochromatic opacity goes through a pronounced minimum at the frequencies corresponding to these overtone bands. Since the Rosseland opacity depends on the inverse of the total opacity, the frequencies at which the minima occur will enter the calculations with the greatest weights. The importance of reliable CIA data in the studies of the protostars seems therefore crucial, especially at the higher frequencies $\left(v=0 \rightarrow v^{\prime}=2,3\right)$ for which model calculations still do not exist. As suggested by Lenzuni \& Saumon (1992) if the inclusion of more accurate CIA predictions of the higher overtones will result 
in a larger opacity, it may profoundly affect the currently established minimum mass of $\mathrm{H}$ burning in the Population III stars.

Finally, Lenzuni \& Saumon (1992) pointed out the likely importance of the many-body effects which are significant at high enough densities. Rough models of these effects have been included and they seem to be important at densities larger than 200 amagats. It needs to be pointed out that whereas spectral moments (integrated intensities) can be estimated for three-body spectra (see, for example, Moraldi \& Frommhold 1989), there is no method yet developed to model spectral densities accounting for the many-body effects, not even for the lowest, 3-body effects in CIA. First attempts in modelling the three body spectral contributions were, however, quite promising (see, for example, Chap. 3, in Frommhold 1993), and efforts in this direction continue.

In general, CIA models should be important also in the modelling of the atmospheres of the low mass zero-metallicity stars, $M$ dwarfs (Allard 1994) and the brown dwarfs (Burrows et al. 1993) with $\mathrm{H}_{2}$-rich atmospheres. Ongoing research (see, for example, Saumon et al. 1994) indicates an increasing interest in collision-induced opacities in the studies of such stellar atmospheres. With more evidence continuously delivered, most likely a greater interest in new CIA spectral contributions will emerge. With it, the need for more calculations, presently not available, is certain to ensue. Even at present, it looks unlikely that the im-

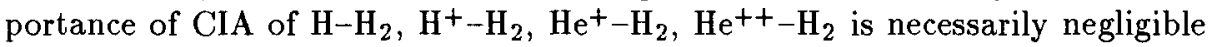
for stars with weakly ionized atmospheres (Yorke 1993).

\section{Quantum Mechanical Computations}

Hydrogen and helium are very light. Even at elevated temperatures, the thermal de Broglie wavelength is still fairly large so that a decidedly quantum mechanical behavior results. For example, at temperatures as high as $7000 \mathrm{~K}$ a wavelength is comparable to that of argon at room temperature, i.e. a few tenths of an Angstrom. As long as the de Broglie wavelength is comparable to the typical intermolecular distances, quantum mechanics, rather than classical theory, has to be used. Moreover, characteristically for all "quantum" systems, only a relatively small number of partial waves (translational orbital angular momen tum) needs to be accounted for in the quantum computations involving $\mathrm{H}_{2}-\mathrm{X}$ pairs. According to the correspondence principle, only the presence of the large quantum numbers indicate a rather "classical" behavior of the system.

As mentioned above, the "binary" spectra, i.e. those due to pairs of molecules (atoms) constitute the major contribution to CIA, at least at low densities ( $\rho \leq$ 10 amagats). The theory for computing quantum mechanical CIA for pairs of molecules is well developed (Birnbaum et al. 1984, Frommhold \& Meyer 1987). The theory is based on the assumption of an isotropic potential. It assumes that for the treatment of molecular dynamics, one can neglect the molecular orientations. Such an approximation simplifies the computations in a fundamental way: rotational and translational motions are decoupled. In that case, the spectra amount to a simple convolution of a well known spectral density function 
of free rotors with the translational profile. It is the latter one where all the computational effort goes: it involves evaluating the induced dipole transition matrix elements between various translational states. For isotropic intermolecular potentials, this requires solving the radial Schrödinger equation, which is by far less complicated than solving a close-coupled set of equations which results when the anisotropy of the system is fully accounted for. The isotropic interaction assumption is quite satisfactory for systems including $\mathrm{H}_{2}$, certainly at low temperatures and if highly vibrational excitation is not involved.

Additionally, induced dipole moments are now available for $\mathrm{H}_{2}-\mathrm{H}_{2}$ and $\mathrm{H}_{2}-\mathrm{He}$ pairs. Whereas for heavy, highly polarizable molecules the multipolar induction approximation gives generally quite agreeable results, it is not sufficient when one deals with $\mathrm{H}_{2}$ and $\mathrm{He}$. State of the art, ab initio quantum computations (Meyer et al. 1989a and Borysow et al. 1990) are fortunately available, which make the computational task reliable.

These highly sophisticated induced dipole surfaces, when combined with the best models of the interaction potential, have been employed in the computations of the rotranslational and rovibrational $\left(v=0 \rightarrow v^{\prime}=0,1,2\right)$ CIA spectra of pure hydrogen and hydrogen-helium mixtures. In each case, remarkable agreement with the measured data (which exist at temperatures below room temperature) has been found, confirming the soundness of both input data and the applied theory. The reader is referred to Meyer \& Frommhold (1989), Meyer et al. (1989b), Borysow et al. (1990) or Meyer et al. (1989a) where such comparisons are presented in full detail. In fact, the success of the method has initiated a long lasting project, which has provided numerous computer models for the planetary scientists involved in atmospheric studies (Borysow \& Frommhold 1991). The case of the high temperatures is somewhat more challenging. Whereas at low temperatures the data can be confirmed by existing measurements, the same cannot be said about the computations for temperatures of $1000 \mathrm{~K}$ and higher. At high temperatures, the input data have to be carefully selected since induced dipole functions at shorter range are significant which are not yet thoroughly tested by measurements. The same caution must be used when an intermolecular potential is used at temperatures much higher than those of experiments. It is in fact the largest source of uncertainty for the theoretical estimates of the high-temperature behavior of the CIA spectra. The numerical accuracy of quantum line shape computations can be kept within the $1 \%$ uncertainty range or so. Under the circumstances, however, it would appear that such computations offer unsurpassed reliability relative to the earlier models if cautiously extended to stellar temperatures. For example, with a given set of input data, the theory provides a temperature dependence of the spectra, which cannot be obtained from any one of the semi-empirical extrapolations proposed heretofore.

\section{Spectral Models for Stellar Atmospheres}

It is desirable that quantum mechanical results are available to the community of interested scientists, over a wide range of temperatures and frequencies. For 
the modelling of atmospheres, one would like to have a quick access to accurate predictions. For that purpose, we have presented our numerical quantum profiles in the form of spectral "model lineshapes". The exact computational results have been fitted to carefully selected analytical model profiles as functions of temperature and frequency, making sure that the accuracy of the original data is not lost (see, for example, Borysow \& Frommhold 1991, Moraldi et al. 1988 or Borysow et al. 1987).

These analytical profiles are extensively tested and match the quantum mechanical profiles within a few percent over the entire molecular band of concern (see, for example, Borysow et al. 1989). Fortran programs exist (see $\mathrm{BF}^{2-4}$, Table 1) which utilize these simple lineshapes to generate reliable CIA data over a huge range of temperatures and frequencies. These are readily available from the author by e-mail (aborysow@phy.mtu.edu).

Table 1 presents a list of all available high temperature CIA data, new and old. Linsky's (1969) work is denoted JL; RP stands for the $a b$ initio model by Patch (1971). BF denotes models based on quantum mechanical computations: $\mathrm{BF}^{1}$ : Borysow et al. (1988), $\mathrm{BF}^{2}$ : Borysow et al. (1989), BF $\mathrm{BF}^{3}$ : Borysow \& Frommhold (1989) and $\mathrm{BF}^{4}$ : Borysow \& Frommhold (1990). Bullets • indicate hydrogen bands at which reliable input data exist, making the quantum computations of the future CIA models possible at temperatures up to $7000 \mathrm{~K}$.

Table 1. Existing CIA models for stellar applications.

\begin{tabular}{|c|c|c|}
\hline Band & $\mathrm{H}_{2}-\mathrm{H}_{2}$ & $\overline{\mathrm{H}_{2}-\mathrm{He}}$ \\
\hline $\begin{array}{l}0 \rightarrow 0 \\
\text { RT CIA }\end{array}$ & $\begin{array}{l}\mathrm{JL}(600-3000 \mathrm{~K}) \\
-\nearrow 7000 \mathrm{~K}\end{array}$ & $\begin{array}{l}\mathrm{BF}^{1}(40-3000 \mathrm{~K}) \\
-\quad 7000 \mathrm{~K}\end{array}$ \\
\hline $\begin{array}{l}0 \rightarrow 1 \\
\text { RV CIA }\end{array}$ & $\begin{array}{l}\mathrm{JL}(600-3000 \mathrm{~K}) \\
\mathrm{BF}(600-5000 \mathrm{~K}) \\
\mathrm{RP}(300-7000 \mathrm{~K})\end{array}$ & $\mathrm{BF}^{2}(20-7000 \mathrm{~K})$ \\
\hline $\begin{array}{l}0 \rightarrow 2 \\
\text { RV CIA }\end{array}$ & $\begin{array}{l}\mathrm{JL}(600-3000 \mathrm{~K}) \\
\bullet \quad \nearrow 7000 \mathrm{~K}\end{array}$ & $\mathrm{BF}^{3}(20-7000 \mathrm{~K})$ \\
\hline "hot bands" & & $\begin{array}{l}\mathrm{BF}(20-7000 \mathrm{~K}) \\
\text { various bands } \\
v \rightarrow v^{\prime} \\
v, v^{\prime}=\{0,1,2,3\}\end{array}$ \\
\hline
\end{tabular}

The new $a b$ initio computations permit for the first time the most reliable comparison with Linsky's often used semi-empirical models. As mentioned above, most crucial for the accuracy of the quantum computations is the induced dipole and the intermolecular potential functions input. For $\mathrm{H}_{2}$ the assumption of the isotropic interaction is suitable, at least as far as our experience with CIA at low temperatures shows. These essential dipole and potential functions have been examined in a great detail. The theoretical computations agree with all experimental data which exist at temperatures up to $300 \mathrm{~K}$. Up to date, no 
laboratory measurements exist at higher temperatures and greater uncertainty results, for example, due to the choice of the potential model. Nonetheless, it seems that the most recent models must be more reliable than the empirical extrapolation used heretofore.

To present just a few examples, let us focus on selected hydrogen bands. Starting with $\mathrm{H}_{2}-\mathrm{H}_{2}$ pairs and the rotranslational band: a preliminary study by Borysow (1993) shows that whereas Linsky's predictions agree quite well with the quantum computations at $600 \mathrm{~K}$, they overestimate the value of the absorption coefficient compared to the quantum results at $1000 \mathrm{~K}$ by approx. $10 \%$, and underestimate them at $3000 \mathrm{~K}(\sim 40 \%)$. The shape of these spectra agrees reasonably well with state-of-the-art quantum calculations.

In the fundamental band the disagreement is even larger. Figure 4 shows the RV CIA spectra of $\mathrm{H}_{2}$ pairs at $3000 \mathrm{~K}$. The peak intensity is now off by a factor of two at $1000 \mathrm{~K}$ and a factor of four at $3000 \mathrm{~K}$. Moreover, the spectral shape differs somewhat. For comparison, the results of ab initio computations made by Patch (1971) are also plotted. They do not agree with the current model either. A cautionary note is due at this point. An advanced model has been proposed

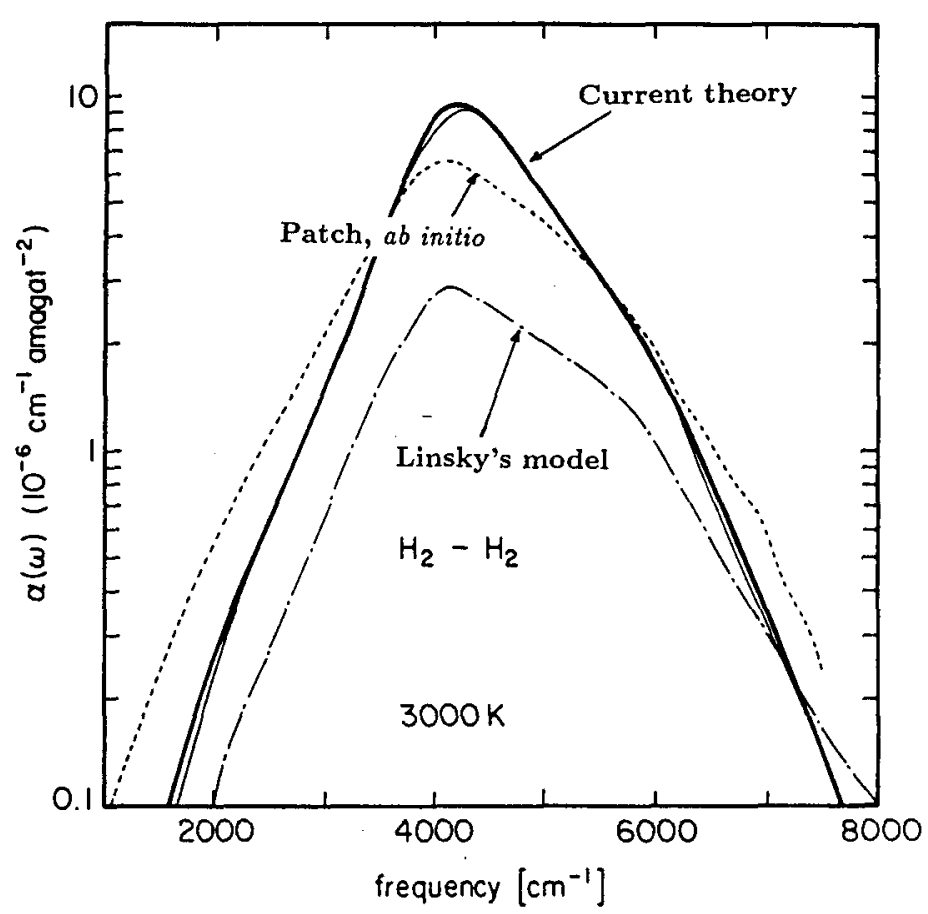

Fig. 4. Rovibrational collision-ind uced absorption spectrum of $\mathrm{H}_{2}-\mathrm{H}_{2}$ at $3000 \mathrm{~K}$ in the fundamental hydrogen band. The solid line represents the current model, reproduced from Borysow \& Frommhold (1990); the dash-dotted line represents for comparison Linsky's (1969) results; the dashed line corresponds to the early calculations by Patch (1971). 
(Borysow et al. 1985), which enables one to reproduce the RT CIA spectra of hydrogen pairs at temperatures below $300 \mathrm{~K}$. This model was never intended to be used at higher temperatures. If one does extend it to stellar temperatures (as, for example, has been done by Lenzuni et al. 1991), the resulting errors amount to as much as a factor of three at temperature of $3000 \mathrm{~K}$ (Borysow 1993), with the current estimates being smaller.

Let us turn next to the discussion of $\mathrm{H}_{2}-\mathrm{He}$ models. For the rotranslational band, Linsky assumed that the spectral shapes of $\mathrm{H}_{2}-\mathrm{H}_{2}$ and $\mathrm{H}_{2}-\mathrm{He}$ are the same. He has rescaled the intensity of the pure hydrogen spectra accounting for the smaller polarizability of helium. This assumption is too crude as the comparisons in Figs. 1 and 2, and in also Fig. 3 show.

The RV CIA spectra of $\mathrm{H}_{2}-\mathrm{He}$ have been computed by Linsky for the fundamental band only. They can be compared with the quantum model (Borysow et al. 1989). We will not show the spectra here. It is enough to mention that the maximum intensity of the two models differs by roughly a factor of four at $3000 \mathrm{~K}$ and a factor of two at $1000 \mathrm{~K}$, with the earlier model predicting too small intensity. Additionally, Linsky's spectra appear as if they were shifted towards the lower frequencies.

\section{Summary}

In the last two decades it has been demonstrated that collision induced absorption may contribute significantly to the opacity of cool stellar atmospheres, predominantly composed of hydrogen and helium. It is now certain that CIA may actually be a dominant source of opacity at low enough temperatures and/or high enough densities. Additionally, it has been pointed out that in such cases the accuracy of CIA intensities used in the atmospheric models may be critical in determining various stellar parameters like, for example, the effective temperature. It is clear that all future model atmospheres of the cool stars like white dwarfs, $M$ dwarfs, brown dwarfs, the protostars, and in general, the zerometallicity, low mass stars, must account for CIA infrared bands of $\mathrm{H}_{2}-\mathrm{H}_{2}$ and $\mathrm{H}_{2}-$ He. Fortunately, reliable and readily available quantum mechanical models exist which can be utilized in the current analyses. Future work should concentrate on modelling of the higher overtones of $\mathrm{H}_{2}-\mathrm{H}_{2}$ absorption, and of the three body spectral contribution to the CIA at high temperatures.

Acknowledgments. The author would like to thank Dr. Lothar Frommhold for careful reading of the manuscript, and his critical comments regarding the presentation of the material.

\section{References}

Allard F., 1994, In: P. Thejll \& U. G. Jørgensen (eds.), Poster session proceedings of IA U Coll. 146, Copenhagen University, p. 1 
Birnbaum G., Chu S.-I., Dalgarno A., Frommhold L., Wright, E. L., 1984, Phys. Rev., 29A, 595

Birnbaum G. (ed.), 1985, Phenomena Induced by Intermolecular Interactions, NATO

ASI Series, B: Physics, (New York: Plenum Press), vol. 127

Borysow A., 1993, unpublished

Borysow A., Frommhold L., 1989, Astrophys. J., 341, 549

Borysow A., Frommhold L., 1990, Astrophys. J. Lett., 348, L41

Borysow A., Frommhold L., 1991, J. Geophys. Res.: Planets, 96, 17,501

Borysow A., Frommhold L., Dore P., 1987, Int. J. of IR and $m m$ Waves, 8, 381

Borysow A., Frommhold L., Moraldi M., 1989, Astrophys. J., 336, 495

Borysow A., Frommhold L., Meyer W., 1990, Phys. Rev., A, 41, 264

Borysow A., Moraldi M., Frommhold L., 1991, In: Trends in Chemical Physics, (India: Council of Scientific Research Integration), 1, p. 83

Borysow J., Frommhold L., 1985, In: G. Birnbaum (ed.), Phenomena Induced by Intermolecular Interactions, (New York: Plenum Press), p. 67

Borysow J., Trafton L., Frommhold L., Birnbaum G., 1985, Astrophys. J., 296, 644

Borysow J., Frommhold L., Birnbaum G., 1988, Astrophys. J., 326, 509

Burrows A., Hubbard W., Lunine J., 1993, Astrophys. J., 345, 939

Frommhold L., 1993, Collision-Induced Absorption in Gases, Cambridge Monographs on Atomic, Molecular and Chemical Physics 2, (New York: Cambridge University Press), 1 edition

Frommhold L., Meyer W., 1987, Phys. Rev., A 35, 632

Frommhold L., Keto J. (eds.), 1990, Spectral Line Shapes, (New York: American Institute of Physics, AIP), volume 6

Lewis J. C., 1985, In: G. Birnbaum (ed.), Phenomena Induced by Intermolecular Interactions, (New York: Plenum Press), p. 215

Lenzuni P., Chernoff D. F., Salpeter E. E., 1991, Astrophys. J. Suppl, 76, 759

Lenzuni P., Saumon D., 1992, Rev. Mexicana Astron. Astrof., 23, 223

Linsky J. L., 1969, Astrophys. J., 156, 989

Meyer W., Frommhold L., 1989, Phys. Rev., A 34, 2771

Meyer W., Borysow A., Frommhold L., 1989, Phys. Rev., A, 40, 6931

Meyer W., Frommhold L., Birnbaum G., 1989, Phys. Rev. A, 39, 2434

Moraldi M., Borysow A., Frommhold L., 1988, Phys. Rev., A, 38, 1839

Moraldi M., Frommhold L., 1989, Phys. Rev., A, 40, 6260

Mould J., Liebert J., 1978, Astrophys. J., 226, L29

Palla F., 1985, In: G. H. F. Diercksen et al. (eds.), Molecular Astrophysics, D. Reidl Publ. Co., p. 687

Patch R. W., 1971, J. Quant. Spectr. and Rad. Transfer, 11, 1331

Saumon D., Bergeron P., Lunine J. I., 1994, In: P. Thejll \& U. G. Jørgensen (eds.), Poster session proceedings of IAU Coll. 146, Copenhagen University, p. 98

Shipman H. L., 1977, Astrophys. J., 213, 138

Stahler S. W., Palla F., Salpeter E. E., 1986, Astrophys. J., 302, 590

Szudy J. (ed.), 1989, Spectral Line Shapes, volume 5. Ossolineum Publishing House

Trafton L. M., 1964, Astrophys. J., 140, 1340

Trafton L. M., 1966, Astrophys. J., 146, 558

Tsuji T., 1969, In: S.S.Kumar (ed.), Low Luminosity Stars, (New York: Gordon and Breach Science Publ.), p. 457

van Kranendonk J., 1968, Can. J. Phys., 46, 1173

Yorke H., 1993, Private communication 